\title{
Experimental Identification of the Kink Instability as a Poloidal Flux Amplification Mechanism for Coaxial Gun Spheromak Formation
}

\author{
S. C. Hsu* and P. M. Bellan \\ California Institute of Technology, Pasadena, California 91125, USA
}

(Received 24 February 2003; published 30 May 2003)

\begin{abstract}
The magnetohydrodynamic kink instability is observed and identified experimentally as a poloidal flux amplification mechanism for coaxial gun spheromak formation. Plasmas in this experiment fall into three distinct regimes which depend on the peak gun current to magnetic flux ratio, with (I) low values resulting in a straight plasma column with helical magnetic field, (II) intermediate values leading to kinking of the column axis, and (III) high values leading immediately to a detached plasma. Onset of column kinking agrees quantitatively with the Kruskal-Shafranov limit, and the kink acts as a dynamo which converts toroidal to poloidal flux. Regime II clearly leads to both poloidal flux amplification and the development of a spheromak configuration.
\end{abstract}

PACS numbers: 52.55.Ip, 52.30.Cv, 52.35.Py

The spheromak $[1,2]$ is a simply connected plasma configuration in which the magnetic fields are largely determined by dynamo-driven plasma currents. Because of its topological simplicity and ease of formation, the spheromak is of interest as a magnetic fusion confinement scheme [3]. Spheromak formation has traditionally been explained by Taylor's hypothesis [4] that a turbulent magnetohydrodynamic (MHD) system relaxes to a state of minimum magnetic energy subject to the constraint of constant magnetic helicity. While this hypothesis has successfully explained the existence and many equilibrium properties of spheromaks, it says nothing about the actual 3D dynamics underlying the relaxation process, e.g., for coaxial gun spheromaks, the dynamo mechanism which converts injected toroidal flux into required poloidal flux [5]. The dynamics must be 3D because Cowling's theorem [6] shows that purely axisymmetric processes cannot accomplish this. This Letter experimentally identifies the MHD kink instability [7] as a 3D mechanism which converts toroidal to poloidal flux in a coaxial gun system, thereby leading to spheromak formation. This mechanism should also be of fundamental importance to coaxial helicity injection in spherical tori [8], relaxation in reversed-field pinches [4], solar coronal plasma instabilities [9], and astrophysical jets [10].

Plasmas in this experiment fall into three regimes depending on peak $\lambda_{\text {gun }}=\mu_{0} I_{\text {gun }} / \psi_{\text {gun }}$ (where $I_{\text {gun }}$ is the gun current and $\psi_{\text {gun }}$ is the bias poloidal magnetic flux intercepting the inner gun electrode), with (I) low values resulting in a straight plasma column with helical magnetic field along the symmetry axis, (II) intermediate values leading to kinking of the column axis, and (III) high values leading immediately to a detached plasma with $B_{\text {tor }} \gg B_{\text {pol }}$. Onset of column kinking agrees quantitatively with the Kruskal-Shafranov limit [7], and the kink acts as a dynamo which converts toroidal to poloidal flux. Regime II clearly leads to both poloidal flux amplification and magnetic field profiles consistent with spheromak formation. These results are qualitatively consistent with recent resistive MHD numerical simulations of electrostatically driven spheromaks [11].

Early coaxial gun experiments [12] demonstrated poloidal flux amplification, and it was postulated that this was due to an observed helical instability. More recently, toroidal mode number $n=1$ instabilities in coaxial gun experiments were reported by several spheromak research groups [13-17] in studies of relaxation during sustainment. Two hypotheses were proposed to explain the instability: (1) development of a $q=1$ surface in the closed-flux region $[13,15]$ or equivalently a magnetic axis kink, and (2) current-driven destabilization of the central column $[16,17]$ or equivalently a geometric axis kink. Additionally, the instability was shown to couple power from the central column to the spheromak [14]. This work offers a significant new result in that a nonlinear $n=1$ geometric axis kink is directly observed to produce the dynamo and poloidal flux amplification leading to spheromak formation.

Building on a prior Caltech spheromak formation experiment [18], the present experiment simplifies the spheromak formation process by using a novel coaxial gun [19], shown in Fig. 1. The gun consists of two concentric, coplanar copper electrodes: a $20 \mathrm{~cm}$ diameter disk (blue) biased to negative polarity high voltage surrounded by a $50 \mathrm{~cm}$ outer diameter annulus (green) connected to vacuum chamber ground. The width of the gap between electrodes is $6 \mathrm{~mm}$. The inner electrode is mounted on the end of a vacuum reentrant port; both are electrically insulated from the vacuum chamber by a ceramic break. The coplanar arrangement of the electrode surfaces is geometrically simple, allows the spheromak formation process to be diagnosed with no mechanical obstructions, and is feasible to model numerically [20]. Bias field poloidal flux $\psi$ contours are also shown in Fig. $1 ; \psi_{\text {gun }}$ can be adjusted from $0-7 \mathrm{mWb}$. Both $I_{\text {gun }}$ and bias field point toward the inner electrode. 


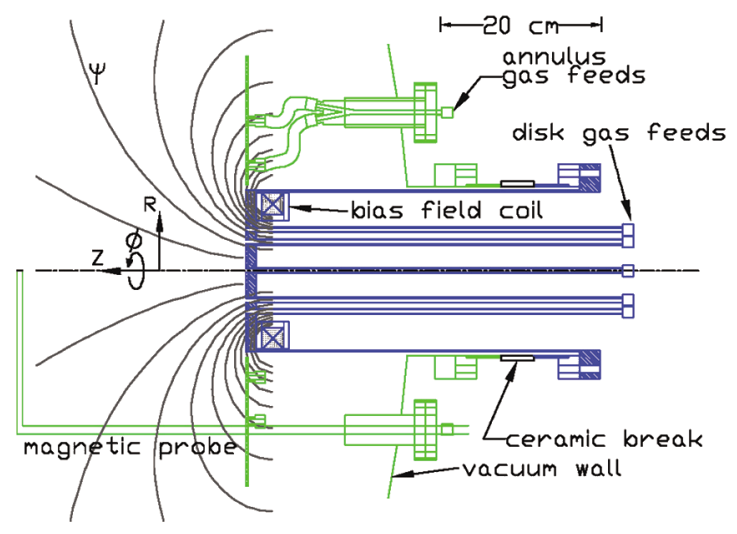

FIG. 1 (color). Schematic of coaxial gun, showing bias field coil, $\psi$ contours, gas feeds, and magnetic probe.

The rise time of $\psi_{\text {gun }}$ is $10 \mathrm{~ms}$, making it essentially stationary on the much faster time scale of the gun discharge (tens of $\mu \mathrm{s}$ ). The gun is installed at one end of a much larger vacuum chamber (about $2 \mathrm{~m}$ long and $1.5 \mathrm{~m}$ diameter), and thus boundary effects on the spheromak formation process are minimized. The gun is powered by an ignitron-switched $120 \mu \mathrm{F}, 20 \mathrm{kV}$ capacitor bank. Hydrogen gas is injected transiently using fast puff valves at eight equally spaced toroidal positions on each electrode. Because of the Paschen effect, the optimum path for plasma breakdown is along the bias field and not at the gap between electrodes. The capacitor bank is discharged at $t=0$, at which time the bias field and gas puff have already been introduced, and breakdown occurs at approximately $4 \mu \mathrm{s}$.

The main diagnostics are a multiple-frame fast chargecoupled device (CCD) camera and a 60-channel magnetic probe array. The camera takes a sequence of timeresolved images in one plasma shot. The interframe time is typically 1 or $1.5 \mu \mathrm{s}$, and the exposure time of each frame is 10 or $20 \mathrm{~ns}$. False color is applied to the images for viewing. The magnetic probe array, shown in Fig. 1, measures all three components $(R, \phi, Z)$ of $\mathbf{B}$ at 20 radial positions with $2 \mathrm{~cm}$ radial spacing. Probe $\dot{B}$ signals are acquired using a digital acquisition system and integrated numerically. For all $B$ measurements in this paper, the probe is located at $Z=22 \mathrm{~cm}$ from the plane of the gun electrodes. Kink occurrence is independent of the probe (see Figs. 2 and 3). Propagation of the plasma in $Z$ past the stationary probe relates temporal information to $Z$-spatial information. This relationship improves as the propagation past the probe becomes fast compared to the plasma expansion rate; this was exploited in the prior Caltech spheromak experiment [18]. The time dependence thus acts as a proxy for the $Z$ dependence. $I_{\text {gun }}$ is measured with a Rogowski coil surrounding the ceramic break, and gun voltage $V_{\text {gun }}$ is measured using a highvoltage probe. Typical parameters are $V_{\text {gun }}=4-6 \mathrm{kV}$ (charge voltage) and 2-2.5 kV (after breakdown), peak

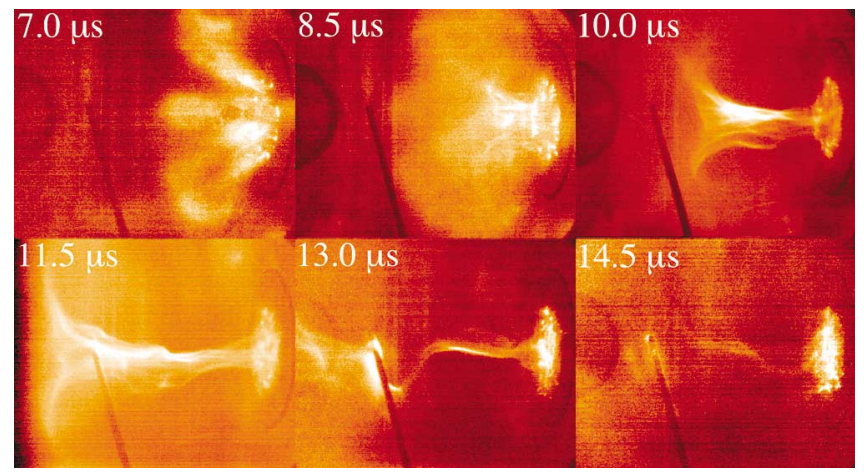

FIG. 2 (color). Image sequence of shot 2472 (DRS Hadland Imacon 200 CCD camera). The circular gap between outer and inner electrodes is visible toward right side of each frame. $B$ probe is also visible. Kink is fully developed by $13 \mu \mathrm{s}$.

$I_{\text {gun }}=70-120 \mathrm{kA}, \quad \psi_{\text {gun }}=0.5-2 \mathrm{mWb}, \quad B \approx 0.1-1 \mathrm{kG}$, $n \sim 10^{14} \mathrm{~cm}^{-3}$, and $T_{\mathrm{e}} \sim T_{\mathrm{i}} \approx 5-20 \mathrm{eV}$.

Figure 2 shows the time evolution of a kinked plasma (shot 2472). Gun electrodes are on the right side of each frame, and the $Z$ axis is oriented horizontally across the middle of each frame. At $7 \mu \mathrm{s}$, bright arches are visible soon after breakdown, showing that breakdown occurs along the vacuum bias field lines. As $I_{\text {gun }}$ increases, the arches expand and quickly merge together $(8.5 \mu \mathrm{s})$, forming a plasma column $(10 \mu \mathrm{s})$ which begins to kink $(11.5 \mu \mathrm{s})$ and then becomes strongly kinked (13 $\mu \mathrm{s})$. It will be shown that this sequence leads to a spheromak. Depending on peak $\lambda_{\text {gun }}$, three distinct plasmas result, as shown in Fig. 3. Regime I leads to a stable column, II to a kinked column and then a spheromak, and III to an immediately detached plasma. The transition from regime I to II (II to III) occurs for peak $\lambda_{\text {gun }} \approx 40(60) \mathrm{m}^{-1}$.

In order to show that the helical perturbation is a MHD kink mode, consider the time evolution of the $q(R)$ profile (relative to geometric axis), which is shown in Fig. 4 (shot 2472). The kink mode becomes linearly unstable when $q(R)=2 \pi R B_{\mathrm{Z}} / L B_{\phi}=1$, where $L$ is the column length; this is the Kruskal-Shafranov limit. Since the kink involves a shift of the current channel, this requires $q=1$ on axis and in its vicinity. As seen in Fig. 4, $q$ near the axis is greater than unity at $9.5 \mu$ s but flattens and

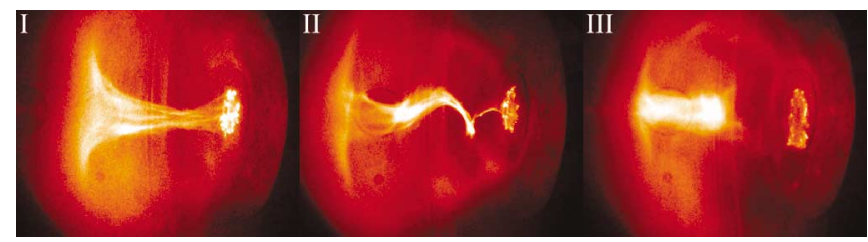

FIG. 3 (color). Images of three plasma regimes which depend on increasing value of $\lambda_{\text {gun }}$ (Cooke Co. HSFC-PRO CCD camera): (I) stable column (shot 1210), (II) kinked column (shot 1247), and (III) detached plasma (shot 1181). $B$ probe was not installed for these shots. 


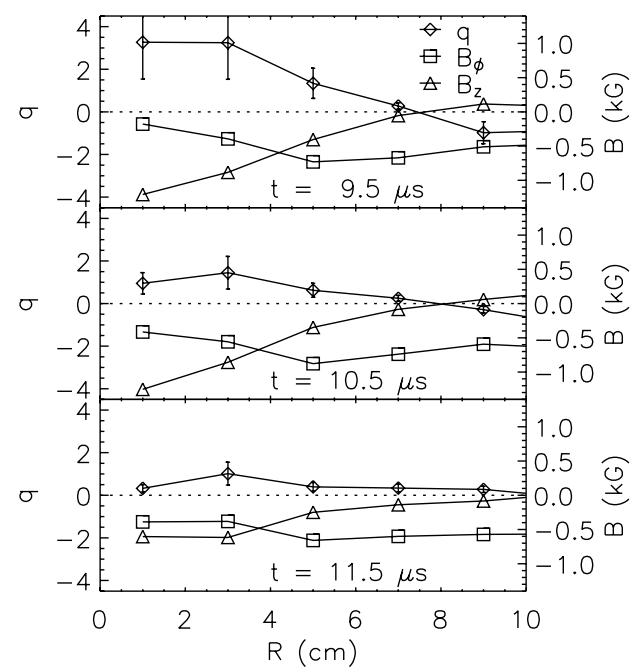

FIG. 4. Flattening of $q(R)$ profile toward unity (shot 2472) right before kink onset. Error bars are due to uncertainty in $L$. Also shown are $B_{\phi}$ and $B_{\mathrm{Z}}$ profiles.

approaches unity by $11.5 \mu \mathrm{s}$, coinciding with the onset of column kinking (Fig. 2). The kink is fully developed at $13 \mu \mathrm{s}$ and has broken apart by $14.5 \mu \mathrm{s}$. The $q$ profile is determined using local $B_{\mathrm{Z}}$ and $B_{\phi}$ data from the magnetic probe array, and $L$ is determined from the CCD images. In addition, for an ensemble of plasma shots in which a column forms (regimes I and II), the Kruskal-Shafranov limit (recast [21] as $\lambda_{\text {gun }}=4 \pi / L$ ) is a good predictor [21] of whether a kink actually develops (Fig. 5). Thus, two sets of independent data (magnetic probe measurements of $B_{\mathrm{Z}}$ and $B_{\phi}$, and Rogowski/flux loop measurements of $\lambda_{\text {gun }}$ ) both give direct evidence that the helical perturbation is a MHD kink mode. It is interesting to note that the observed kinks always have one axial wavelength, even though there is no fixed boundary at the end of the plasma column. One explanation [22] for this is that there is a strong axial gradient in $B n^{-1 / 2}$, and thus an effective Alfvén speed discontinuity, which acts as a rigid boundary. This conjecture is consistent with the "mushroom cap" on the left side of the kink in Fig. 3.

Next, it is shown that the kink is followed immediately by three signatures of spheromak formation: (1) appearance of closed $\psi$ contours [calculated assuming axisymmetry, $\left.\psi(R, t)=\int_{0}^{R} 2 \pi R^{\prime} B_{\mathrm{Z}}\left(R^{\prime}, t\right) d R^{\prime}\right]$, (2) $\psi$ amplification, and (3) magnetic field radial profiles consistent with spheromak formation. It is important to note that the relationship between closed $\psi$ contours and closed-flux surfaces becomes ambiguous when axisymmetry is broken. Thus, in the presence of a nonaxisymmetric rotating kink, closed $\psi$ contours indicate closed-flux surfaces only in a time-averaged way. Shortly after $13 \mu \mathrm{s}$, the kink breaks apart (Fig. 2). This coincides with signatures of spheromak formation as observed in the magnetic probe measurements (Fig. 6). At approximately $13 \mu \mathrm{s}$, closed $\psi$ contours appear

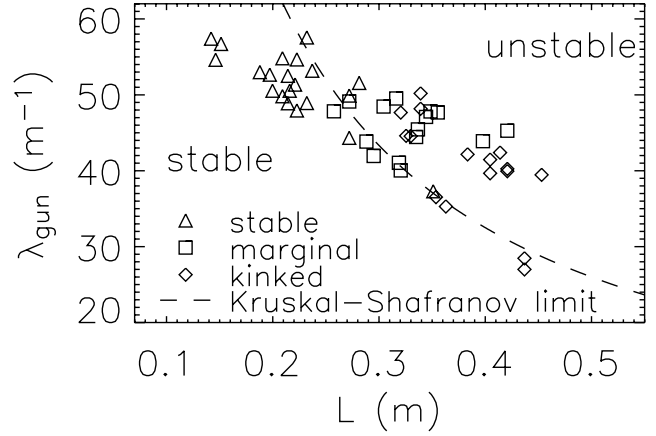

FIG. 5. Plot of $\lambda_{\text {gun }}$ vs column length $L$, showing agreement with Kruskal-Shafranov limit.

and $\psi_{\max }$ is amplified to larger than $\psi_{\text {gun }}(\psi$ amplification is due mainly to broadening of the $B_{\mathrm{Z}}$ profile after $12 \mu \mathrm{s}$ ). At $15 \mu \mathrm{s}$, magnetic field profiles consistent with spheromak formation are observed (Fig. 7). The measured radial profiles of $B_{\mathrm{Z}}$ and $B_{\phi}$ at $15 \mu \mathrm{s}$ are compared with Taylor state solutions [4] in cylindrical geometry, i.e., uniform $\lambda$ solutions of

$$
\nabla \times \mathbf{B}=\lambda \mathbf{B} .
$$

The solutions are $B_{\mathrm{Z}} \sim J_{0}(\lambda R)$ and $B_{\phi} \sim J_{1}(\lambda R)$, where $J_{0}$ and $J_{1}$ are Bessel functions of order zero and one, respectively, and the best fit is found for $\lambda \approx 15 \mathrm{~m}^{-1}$ with a radial offset of $4 \mathrm{~cm}$ (displacement of spheromak off the geometric axis). The slight disagreement between measured profiles and the Taylor solution is not surprising since the spheromak is expected to be either (1) still undergoing relaxation toward the Taylor state or (2) in a modified relaxed state since it is still being driven by the gun which has peak $\lambda_{\text {gun }} \approx 50 \mathrm{~m}^{-1}$.

The kink modifies the direction of current flow from $Z$ (poloidal) to $\phi$ (toroidal), as seen in Fig. 2. Equivalently,

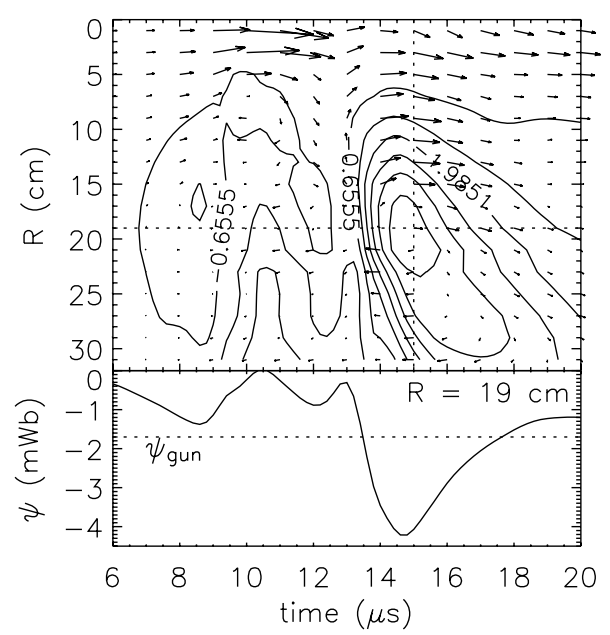

FIG. 6. Plots (shot 2472) vs $R$ and time of (top) $B_{\text {pol }}$ vectors and $\psi$ contours $(\mathrm{mWb})$, and (bottom) $\psi$ along horizontal dashed line of top panel. 


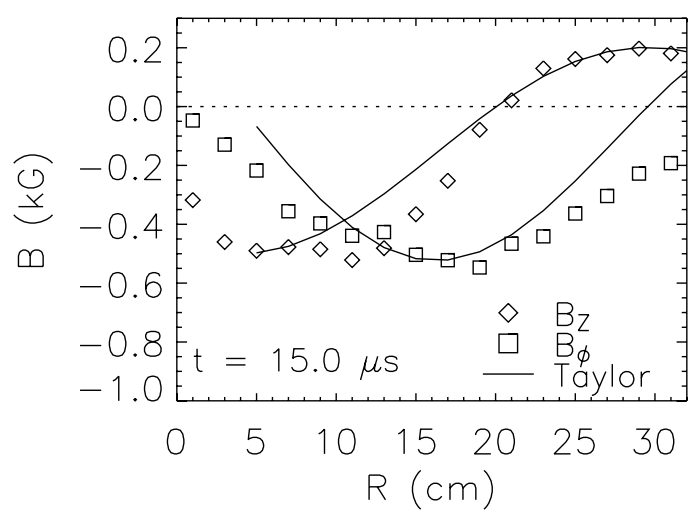

FIG. 7. Plots showing $B_{\mathrm{Z}}, B_{\phi}$ profiles (shot 2472) compared to Taylor solutions $\left(\lambda=15 \mathrm{~m}^{-1}\right.$ and radial offset of $\left.4 \mathrm{~cm}\right)$. Profiles are plotted for vertical dashed line in Fig. 6 (top).

it converts toroidal to poloidal flux. This process is paramagnetic, amplifying $\psi$ over the initial applied $\psi_{\text {gun }}=$ $1.7 \mathrm{mWb}$. The paramagnetism is understood by realizing that kinks involve perturbations with dependence $\exp (i \mathbf{k} \cdot \mathbf{x})$ where $\mathbf{k} \cdot \mathbf{B}=0$. The latter means $k_{\mathrm{Z}}=$ $-n B_{\phi} / R B_{Z}$. Thus, a locus of constant phase of the kink is given by $\phi=\left(B_{\phi} / R B_{Z}\right) Z$, meaning that the kinked current channel is a right (left) handed helix if $J_{\mathrm{Z}} B_{\mathrm{Z}}>0$ $(<0)$. This will always lead to amplification of the original $B_{Z}$. The additional $\psi$ introduced by the kink can be estimated by approximating the helix as a solenoid with current $I$, turns per length $1 / L$, and radius $a$. The solenoid formula for the field inside the solenoid is $B_{\mathrm{Z}}=\mu_{0} I / L$; the $\psi$ produced by the solenoid is $\pi a^{2} B_{\mathrm{Z}}$ and thus depends nonlinearly on the kink amplitude $a$. Using the measured values $a \approx 5 \mathrm{~cm}, L \approx 20 \mathrm{~cm}$, and $I \approx 60 \mathrm{kA}$ at $13.5 \mu \mathrm{s}$, the $\psi$ generated by the kink is predicted to be approximately $1 \mathrm{mWb}$, which is within a factor of 2 of the observed amplification of $\psi_{\max }$ over $\psi_{\text {gun }}$. The discrepancy is within the accuracy of $a$ and $L$ measurements and of the $\psi$ calculation assuming axisymmetry in the presence of the rotating kink. Because the coaxial gun can inject only toroidal flux, 3D plasma dynamics must be responsible for $\psi$ exceeding $\psi_{\text {gun }}$. The dynamics are provided by the kink, which amplifies $\psi_{\text {gun }}$ by converting toroidal to poloidal flux; thus, in this case, the kink constitutes the dynamo intrinsic to spheromak formation.

Regime II clearly leads to all three signatures of spheromak formation: closed $\psi$ contours, $\psi$ amplification, and proper magnetic field profiles. Regime I lacks both closed $\psi$ contours and $\psi$ amplification. Regime III may have closed $\psi$ contours; however, $B_{\text {tor }} \gg B_{\text {pol }}$ associated with high $I_{\text {gun }}$, which could indicate that the plasma is still relaxing. This work indicates a close relationship between the kink threshold $\left(\lambda_{\text {gun }}=4 \pi / L\right)$ and the spheromak formation threshold, which according to a static forcefree treatment is also proportional to an inverse length [2]. Geometric differences among different experiments could result in different eigenmode spectra of Eq. (1) relative to kink thresholds.

In summary, the MHD kink instability has been identified experimentally as a poloidal flux amplification mechanism for coaxial gun spheromak formation. An $n=1$ central column helical instability was observed during formation using multiple-frame CCD imaging. Onset of the perturbation was shown using two independent sets of data to agree quantitatively with the Kruskal-Shafranov limit. The kink acts as a dynamo which converts toroidal to poloidal flux, and it is followed immediately by three key signatures of spheromak formation: (1) closed $\psi$ contours, (2) $\psi$ amplification, and (3) magnetic field profiles similar to the Taylor state.

The authors acknowledge S. Pracko, C. RomeroTalamás, and D. Felt for technical assistance and $\mathrm{H}$. Bindslev for asking about kink paramagnetism. This work was supported by U.S.-DOE Grant No. DE-FG0398ER544561 and Fusion Energy Sciences Fellowship Program.

*Current address: P-24 Plasma Physics Group, Los Alamos National Laboratory, Los Alamos, NM 87545, USA.

[1] T. R. Jarboe, Plasma Phys. Controlled Fusion 36, 945 (1994).

[2] P. M. Bellan, Spheromaks (Imperial College Press, London, 2000).

[3] H. S. McLean et al., Phys. Rev. Lett. 88, 125004 (2002).

[4] J. B. Taylor, Rev. Mod. Phys. 58, 741 (1986).

[5] A. al-Karkhy et al., Phys. Rev. Lett. 70, 1814 (1993).

[6] T. G. Cowling, Mon. Not. R. Astron. Soc. 94, 39 (1934).

[7] J.P. Freidberg, Ideal Magnetohydrodynamics (Plenum Press, New York, 1987).

[8] R. Raman et al., Phys. Rev. Lett. 90, 075005 (2003).

[9] B. Vršnak, V. Ruždjak, and B. Rompolt, Sol. Phys. 136, 151 (1991).

[10] H. Li et al., Astrophys. J. 561, 915 (2001).

[11] C. R. Sovinec, J. M. Finn, and D. del-Castillo-Negrete, Phys. Plasmas 8, 475 (2001).

[12] L. Lindberg and C.T. Jacobsen, Phys. Fluids 7, S44 (1964).

[13] S. O. Knox et al., Phys. Rev. Lett. 56, 842 (1986).

[14] P. K. Browning et al., Phys. Rev. Lett. 68, 1718 (1992).

[15] M. Nagata et al., Phys. Rev. Lett. 71, 4342 (1993).

[16] R. C. Duck et al., Plasma Phys. Controlled Fusion 39, 715 (1997).

[17] D. Brennan et al., Phys. Plasmas 6, 4248 (1999).

[18] J. Yee and P. M. Bellan, Phys. Plasmas 7, 3625 (2000).

[19] S. C. Hsu and P. M. Bellan, IEEE Trans. Plasma Sci. 30, 10 (2002).

[20] M. Tokman, S. C. Hsu, and P. M. Bellan, Bull. Am. Phys. Soc. 47, 42 (2002).

[21] S. C. Hsu and P. M. Bellan, Mon. Not. R. Astron. Soc. 334, 257 (2002).

[22] M. Nakamura, Y. Uchida, and S. Hirose, New Astron. 6, 61 (2001). 